\title{
Review of: "Protein interface redesign facilitates the transformation of nanocage building blocks to 1D and 2D nanomaterials"
}

\author{
Kazunori Matsuura ${ }^{1}$ \\ 1 Tottori University \\ Potential competing interests: The author(s) declared that no potential competing interests exist.
}

This paper clearly demonstrated that redesign of protein-protein interactions at the interfaces of dimeric Thermotoga maritima ferritin (TmFtn) proteins rendered the self-assembly transformation from original nanocage to filament, nanorod and nanoribbon by responding external stimuli. Although transformation of supramolecular morphologies of artificial protein assemblies remains challenging issue, the authors revealed with high accuracy TEM, AFM, and X-ray data that the rational mutation of interface of TmFtn dimer facilitates the morphological transformation. I recognize this study have an impact on the future direction in the fields of protein engineering, nanomaterial science, and supramolecular chemistry. But, there are some concerns with this study as follows:

1. Does the morphology of protein assemblies depend on the protein concentration? Especially, when the concentration of FLAL (fully side-by-side assembly) is significantly increased, does the morphology change from nanorod to nanoribbon in the presence of PEG?

2. What is the essential role of PEG in the morphological change of the protein assemblies? Is it a depletion interaction due to the excluded volume effect of PEG or a template effect due to the direct interaction between PEG and protein?

3. How important is the p-p stacking interactions of F147 in the self-assembly of FLALs? Looking at the crystal structure in Figure 3C, the two benzene rings do not appear to overlap. What happens to selfassembly behavior when F147 is replaced with another hydrophobic amino acid (such as L or V)?

4. In supplementary Figure 19, AFM image revealed that the height of the nanoribbon is nearly $10 \mathrm{~nm}$, corresponding to about 5 layers of FLAL-L assemblies. The authors should explain why was only 5 layers observed. Why is not the stepped layer image observed?

5. In my opinion, it would be more interesting if a kind of mutant of TmFtn could be used for reversible (dynamic) morphological transformation of the protein assembly of by external stimuli such as $\mathrm{pH}$, ions, and light. If possible, I hope the authors to challenge it in future research. 